\title{
Toward Robotized Beating Heart TECABG: Assessment of the Heart Dynamics Using High-Speed Vision
}

\author{
Loïc Cuvillon ${ }^{1}$, Jacques Gangloff ${ }^{1}$, Michel de Mathelin ${ }^{1}$, \\ and Antonello Forgione ${ }^{2}$ \\ ${ }^{1}$ LSIIT / EAVR (UMR CNRS-ULP) ${ }^{\star}$, Strasbourg I University, France \\ \{loic, jacques, demath\}@eavr.u-strasbg.fr \\ 2 IRCAD / EITS, University Hospital of Strasbourg, France
}

\begin{abstract}
Active robotic filtering is a promising solution for beating heart Totally Endoscopic Coronary Artery Bypass Grafting (TECABG). In this work, we assess the heart motion dynamics using simultaneously high speed imaging of optical markers attached to the heart, ECG signals and ventilator airflow acquisitions. Our goal is to make an assessment of the heart motion (shape, velocity, acceleration) in order to be able to make more accurate specifications for a dedicated robot that could follow this motion in real-time. Furthermore, using the 2 additional inputs (ECG, airflow), we propose a prediction algorithm of the motion that could be used with a predictive control algorithm to improve the tracking accuracy.
\end{abstract}

\section{Introduction}

Beating heart TECABG will probably bring a great improvement in coronary revascularization surgery. Current open surgery techniques on the beating heart make use of mechanical stabilizers to reduce the motion of the working area. But they still need a sternotomy which induces a long recovery period for the patient.

Totally endoscopic stabilizers begin to be commercially available (e.g. the Octopus TE from Medtronics) but due to their long size and the way they are attached, the residual motion is significant. So it seems that the best way to achieve good precision in TECABG on the beating heart is to have an active filtering system e.g. a robot that follows the heart motion in real time. Experiments with robotic prototypes and high speed cameras $(500-1000 \mathrm{~Hz})$ demonstrate the feasibility of active compensation on pig's heart [1] 2. However, the achieved tracking accuracy $(1 \mathrm{~mm})$ remains greater than the minimum accuracy needed for grafting a medium sized coronary artery $(0.1 \mathrm{~mm})$.

From these experiments, it comes out that a better knowledge of the dynamic behavior of the heart motion would greatly improve the design of the system for mainly 3 reasons : 1) the motion of the heart has some interesting properties that should be taken into account to predict its behavior in combination with a

\footnotetext{
* The authors wish to thank the Alsace Regional Council and Centre National de la Recherche Scientifique for the grant which support this research project.
} 
predictive control scheme. 2) a knowledge on how the heart moves (acceleration, amplitude, shape of the cycle) would certainly help defining more accurately the specifications of the dedicated mechanical structure. 3) the main concern in medical robotics is safety, so knowing the behavior of the heart with respect to redundant input signals should improve the robustness of the whole procedure.

Some previous studies have already dealt either with heart motion properties or its modeling. In 2004 3, Cattin et al. assess the significance of the residual motion of a pig's beating heart after stabilization with an Octopus. The repeatability of the stabilized cardiac motions, providing that hemodynamics are constant, is underlined but no model is proposed. In 4, Ortmaier studies robust motion prediction of heart landmarks with ECG and respiration as additional robust landmark. In [5], Fourier coefficients of respiratory and cardiac components are estimated by a two-stage adaptive algorithm. A similar approach based on adaptive filtering to separate the two components and predict the future motion is presented in [1. These frequential models assume that ventilation and heartbeat components are independent and invariant.

In this work, we assess the local motion properties of heart for the free and stabilized case. We take advantage of a high speed vision sensor to measure more precisely heart beating velocity, acceleration and their correlation with the ECG waves and ventilation airflow. The analysis of this data demonstrates that the heart global motion is not the result of two independent components : it shows that the shape of the cardiac cycle depends on the lung volume. Finally, we demonstrate that arrhythmic behavior of the heart can be predicted using ECG signals. In a second part, a model of the heart motion that takes into account this coupling is proposed in order to predict and anticipate future motion with a robotic device. This model, with biological signals as inputs, is then evaluated and compared with the frequential model found in the literature.

\section{Materials and Methods}

Experiments were carried out on a pig that underwent a sternotomy with general anesthesia. After positioning the chest retractor, a coronary artery was stabilized using an Octopus v4.3 tissue stabilizer from Medtronics. A rotating knob allows to modify the stiffness of the Octopus arm and so to free or constraint local motion of the heart.

To measure heart motion, a $500 \mathrm{~Hz}$ high speed camera with a $256^{*} 256$ pixel grayscale sensor (DALSA CAD6) is placed on a tripod, its lens focused on the stabilized area (figure 10. When its arm is free, the Octopus stabilizer can be used as a convenient way to simply attach visual markers to the heart. The pose of the myocardium area of interest is computed in the camera frame of reference using the modified version of the Dementhon algorithm for coplanar points.

ECG signals are acquired with a classical 3-leads cable and the CARDIOVIT AT-6 ECG from Schiller, which outputs analog amplified and filtered ECG sig-

nals. Due to built-in signal pre-processing, it exhibits an average group delay of $8 m s$ between electrodes potentials and the ECG analog outputs. Two AWM700 airflow sensors from Honeywell are used for the real-time measurement of the 

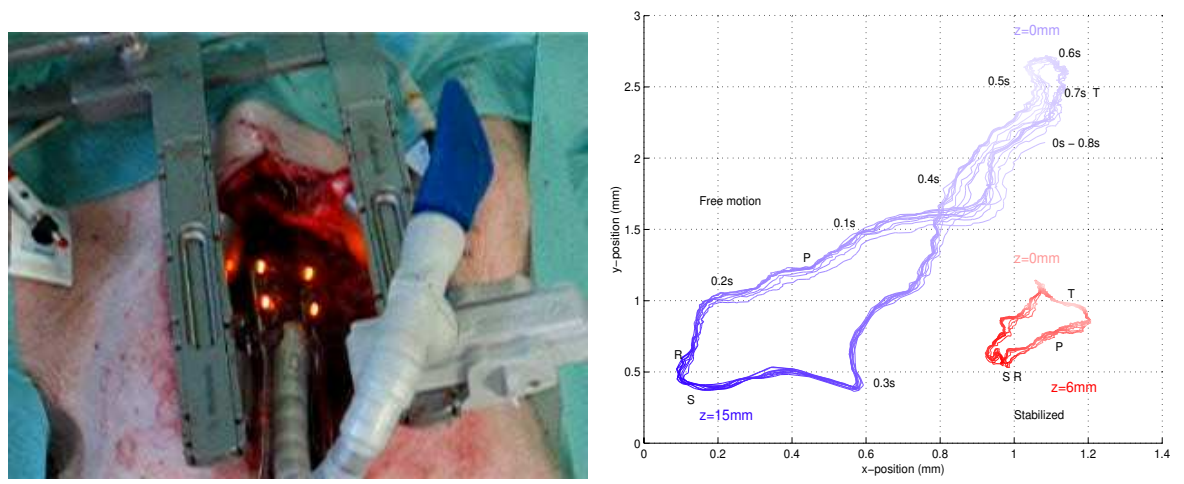

Fig. 1. The heart stabilizer OCTOPUS Fig. 2. Heart trajectories in absence of and its 4 visual markers ventilation with timing and ECG waves

ventilator flow. This uni-directional sensor specially designed for biomedical use has a 6-millisecond response time.

Both ECG signals and airflow measurements are then acquired at $500 \mathrm{~Hz}$ by a PCI acquisition board synchronized with the image acquisition. The whole acquisition software runs on RTAI, a real-time operating system, in order to ensure perfect synchronization and minimal jitter.

\section{Motion of an Area of Interest (AOI) on the Heart}

\subsection{Heart Beating Motion in Absence of Ventilation}

Stabilized residual motion. Trajectories of 10 consecutive heartbeats with and without stabilization are plotted in figure 2. It appears that the excursion range of the AOI is about $2.3 \mathrm{~mm}$ in the frontal plane and $15 \mathrm{~mm}$ in the sagital plane for the free motion case. With a constrained motion (maximum stiffness of the octopus arm), the amplitude of the heart motion is highly damped by the stabilizer: the excursion range is reduced to $500 \mu \mathrm{m}$ and $6 \mathrm{~mm}$ respectively. These values of excursion ranges are similar to those reported in 3 for the residual motion of a stabilized AOI with constant hemodynamics.

Repeatability of the beating trajectory. The repeatability of heart beating trajectory in absence of ventilation and cardiac arrhythmia is a very interesting feature. In the plots of figure 2 for 10 heart beats, the maximum deviation and standard deviation around a mean trajectory on the free and stabilized case are reported in table1. Thus, considering this perfect periodicity, a repetitive control scheme (an example is given in [1]) should give really good results if we assume that respiration can be stopped for a short amount of time. The standard and maximal deviation give then a clue on the accuracy such a system could achieve.

AOI velocity and ECG signal correlation. The heart local velocity is plotted in figure 3. Some markers are added to show the occurrences of T,P,R and S 

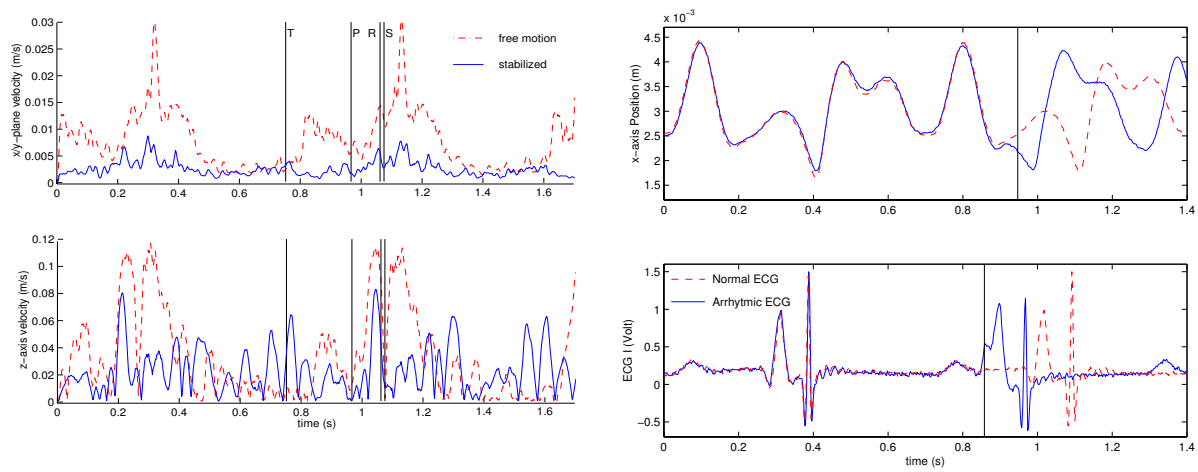

Fig. 3. Heartbeat velocity magnitude with ECG-waves timing in the frontal and heart beating: arrhythmic case sagital planes

Table 1. Deviations with respect to the mean trajectory of 15 consecutive heart beats

\begin{tabular}{|c|c|c|c|c|}
\hline & \multicolumn{2}{|c|}{ frontal plane } & \multicolumn{2}{c|}{ sagital plane } \\
\hline & max deviation & std deviation & $\max$ deviation & std deviation \\
\hline free motion & $146.6 \mu \mathrm{m}$ & $25.6 \mu \mathrm{m}$ & $1.6 \mathrm{~mm}$ & $0.39 \mathrm{~mm}$ \\
stabilized & $63.7 \mu \mathrm{m}$ & $14.7 \mu \mathrm{m}$ & $1.5 \mathrm{~mm}$ & $0.4 \mathrm{~mm}$ \\
\hline
\end{tabular}

waves of the ECG. The cardiac period is $810 \mathrm{~ms}$. Strong accelerations (therefore high velocities) occur $60 \mathrm{~ms}$ after the T, P and QRS complex waves. On the other hand, heart rest (low velocities) covers a range from $200 \mathrm{~ms}$ after the QRS complex to the next $\mathrm{T}$ waves. The maximum acceleration is $0.3 \mathrm{~m} . \mathrm{s}^{-2}$ measured in the frontal plane and $10 \mathrm{~m} . \mathrm{s}^{-2}$ measured in the sagital plane. These values should be taken into account when writing the specifications of an active robotic stabilizer. Moreover, after injection of a high dose of adrenaline, maximum velocities and maximum accelerations were doubled.

In figure 4, we assess the possibility of predicting arrhythmic behavior of the heart. The vertical cursors indicate in both plots the time when the error between arrhythmic ECG (resp. motion) in plain lines and normal ECG (resp. motion) in dashed lines becomes greater than $10 \%$ of the peak to peak amplitude. This figure shows that abnormal ECG can be detected $\approx 90 \mathrm{~ms}$ before the abnormal motion (mean delay $=80 \mathrm{~ms}$, std. deviation $=32 \mathrm{~ms}$ on 12 arrhythmias) letting quite enough time to a robotic system to switch to a failsafe mode.

\subsection{Heart AOI Motion Properties with Ventilation}

Influence of ventilation on heart motion. During respiration, variation of the lung volume yields a motion of the heart within the chest. In figure 5, the motion of a heart AOI and the corresponding lung volume are recorded over a full respiration cycle with the following parameters : a tidal volume set to $600 \mathrm{~mL}$ and a frequency of 21 breaths/min. 


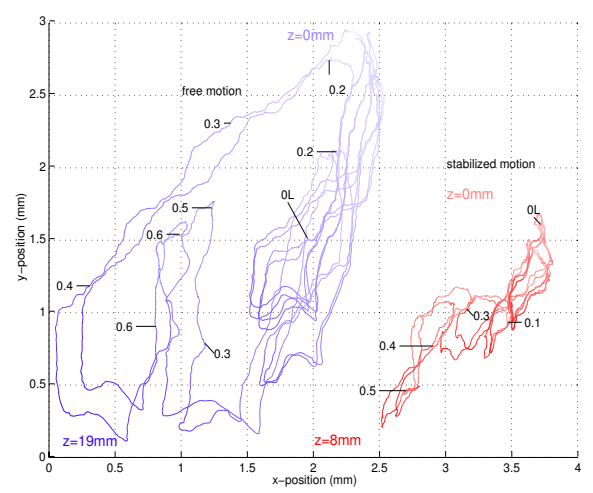

Fig. 5. Heart 3D trajectories with lungs volume information (depth $\mathrm{z}$ coded in color intensity)
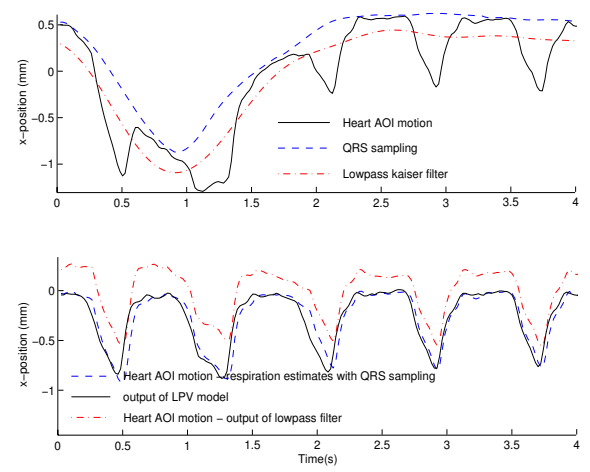

Fig. 6. Respiration and cardiac component over one ventilation period, extracted by proposed model and lowpass filtering

The slow motion component of the heart AOI appears to be directly correlated with the lung volume. Note the different paths followed by the AOI during forced inspiration and the free expiration. Moreover, for low lung volume, i.e. at the end of expiration, the 3D trajectory of the heart AIO during a heart beat is similar to the one observed in total absence of ventilation.

Extraction of the ventilation component. ECG signal acquisition can be used as a clock to sample the heart AOI position when e.g. the heart is 'at rest'. This way it is possible to suppress the heart beating motion and to access to the respiration component alone.

The detection of the QRS complex can be performed on-line by derivation and adaptive thresholding of the ECG signal. From the original ECG signal, a discrete clock signal $Q R S[k]$ with impulses corresponding to QRS detection time is created. In order to increase precision, the previous clock can be delayed by half a cardiac period $\left(T_{b} / 2\right)$ to sample the respiration while the heart is at rest (when the velocity is slow, see fig [3). By interpolating between the respiratory samples, the respiration motion is reconstructed and subtracted from the global motion in order to isolate the heart beating motion.

The upper plot in figure 6 shows the reconstructed respiratory component and the lower plot shows the isolated heart beating component. The beating motion is very similar to the motion in absence of ventilation, however it is modulated by the lung volume. So we cannot assume that the beating is decoupled from respiration.

\section{A Heart Motion Model Based on Biological Signals}

\subsection{Description of the Model}

The proposed model is based on the extraction of the respiration component presented earlier. Both the integrated airflow, so the air volume, and QRS 
occurrences of the ECG signal are used. The proposed algorithm is a 2 stages component identification:

1) In a first step, the profile of the respiration component $r$ is extracted with the approach described in the previous section. Let $k$ be the current sample number and $T_{o v}$ be the sample number corresponding to the beginning of a new respiration cycle. This beginning can be easily detected by a simple thresholding of the volume information. Then :

$$
r[k]=F r\left(k-T_{o v}\right)
$$

where Fr is a spline function which interpolates one period of the respiration component.

2) In a second step, the heart beating component is extracted from the global motion by subtracting the respiration component. As it was shown before, the heart beating depends on lung volume. In order to take into account this coupling, a Linear Parameter Variant (LPV) Finite Impulse Response model is proposed. This model input is an impulse at each QRS occurrences and its parameters depend on lung volume. Let $v[k]$ be the lung volume and $Q R S[k]$ the impulse signal corresponding to the QRS occurrence. The heart position due to cardiac beating $b[k]$ is then given by :

$$
\begin{aligned}
& b[k]=F b(v[k]) Q R S[k-1] \\
& \triangleq\left(\begin{array}{llll}
a_{10}+a_{11} v[k] & a_{20}+a_{21} v[k] & \ldots & a_{d 0}+a_{d 1} v[k]
\end{array}\right)\left(\begin{array}{c}
Q R S[k-1] \\
\vdots \\
Q R S[k-d]
\end{array}\right)
\end{aligned}
$$

where $d$, is the number of samples in one cardiac period. With this formulation, the coefficients $a_{i 0}$ represent the mean value of the beating position, $i$ samples after the previous QRS complex and $a_{i 1}$ is the variation of this position related to lung volume. Identification of the coefficients $\left\{\left(a_{i 0}, a_{i 1}\right), i=1 \ldots d\right\}$ can be performed online via a recursive least mean square (RLS) algorithm for LPV systems, where the criterion of minimization is the error $e[k]$ between the model output and the reference signal [6].

This identification scheme is summarized in the upper part of the figure 7 The global motion $h[k]$ of the heart AOI at sample $k$ is given by :

$$
\begin{aligned}
h[k] & =r[k]+b[k] \\
& =F r\left(k-T_{o v}\right)+F b(v[k]) Q R S[k-1]
\end{aligned}
$$

\subsection{Validation and Comparison with other Approaches}

The output of the proposed model is used to predict the motion of the heart some steps ahead. We compare the accuracy of these predictions with respect to other methods found in the literature. We use a set of data down-sampled at $50 \mathrm{~Hz}$ with a ventilation frequency of $f_{r}=0.242 \mathrm{~Hz}$ and a cardiac frequency $f_{b}=1.23 \mathrm{~Hz}$. We predict the motion $\Delta=T_{b} / 2=20$ samples ahead, so $1 / 2$ heart beat ahead. 

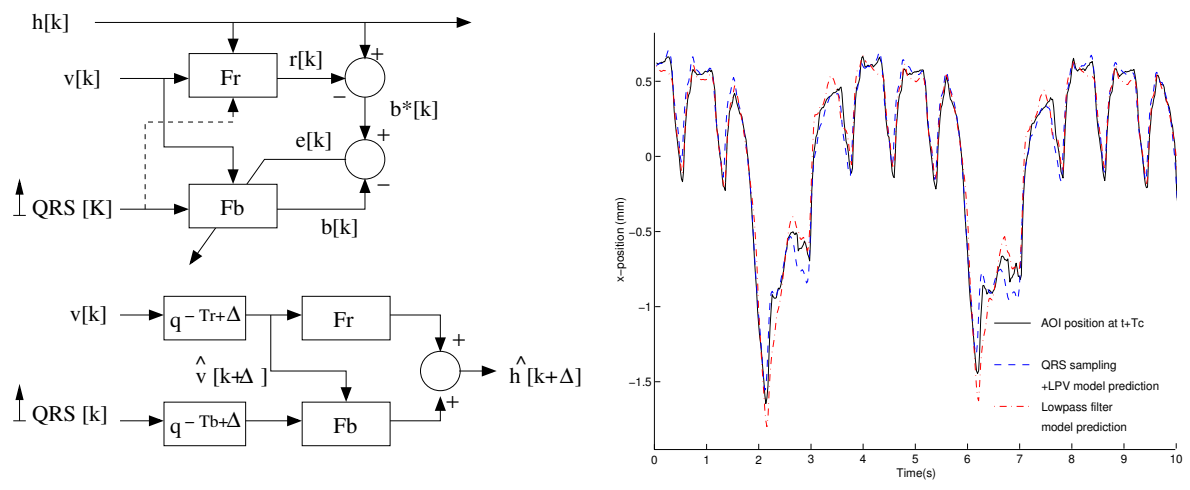

Fig. 7. Identification of the respiration Fig. 8. Comparison of predictions with the component using QRS sampling method lowpass filter approach and with the LPV (top) and the corresponding prediction al- approach gorithm (bottom)

On one hand, the classical frequential approach filters the global motion to extract the respiration component. One can use a lowpass filter or adaptive filter [1], or also Fourier coefficient estimation [5] to suppress heart beating harmonics W].th equivalent results. In this study, a kiser-FIR lowpass filter with a cut-off frequency located between the fundamental of the beating $f b$ and the last significant harmonic of the respiration $(4 f r)$ is used to separate the 2 components. Predicting $\Delta$ steps in the future is then done with pure delays $\left(q^{-1}\right.$ operator) since the components are equal to themselves in the past with the assumption of perfect decoupling and quasi-periodicity.

On the other hand, in our approach, the prediction assumes only that biological signals (QRS complex and ventilation parameters) remain quasi-periodic over the next beating period (lower part of the figure 7 ). In figure 6 , the respiration component extracted from the global motion with the two methods are plotted on the top whereas cardiac motion resulting from the LPV model output or from the lowpass filter output are presented on the bottom.

Finally, predictions of heart motion $\Delta$ steps ahead for both strategies are shown on the figure 8 . The continuous line plots the real heart motion $\Delta$ steps in the future, whereds the other curves give the predictions with the 2 compared method. Standard deviation error of the prediction is $131 \mu m$ ( $4.7 \%$ of the total amplitude) in the frontal plane and $530 \mu(2.8 \%)$ in the sagital plane for our proposed method. For comparative purpose, standard deviation of the error is respectively of $152 \mu \mathrm{m}(5.5 \%)$ in the frontal plane and $547 \mathrm{um}(2.9 \%)$ for the lowpass approach. If we compare the errors in the image plane (this is relevant when doing image-based visual servoing as in [1]), the standard deviation of the prediction error is 0.62 pixels along $x$ and (0.78 pixels along $y$ when using lowpass filtering whereas it falls to 0.37 and 0.41 pixels respectively with the LPV technique. 


\section{Discussion}

This study shows that there is a coupling between the motion components of the heart : the shape of the beating is modulated by the state of the respiration. Furthermore, we measured that the cardiac beating component exhibits very sharp transients (up to $2 g$ acceleration). We propose a new motion prediction algorithm using LPV techniques that takes into account this coupling. This approach improves prediction accuracy.

\section{References}

1. Ginhoux, R., Gangloff, J., de Mathelin, M., Soler, L., Sanchez, M.A., Marescaux, J.: Active filtering of physiological motion in robotized surgery using predictive control. IEEE Transactions on Robotics 21 (2004) 67-79

2. Nakamura, Y., Kishi, K., Kawakami, H.: Heartbeat synchronization for robotic cardiac surgery. In: Proc. of the IEEE International Conference on Robotics and Automation, Seoul, Korea (2001)

3. Cattin, P., Dave, H., Grünenfelder, J., Szekely, G., Turina, M., Zund, G.: Trajectory of coronary motion and its signicance in robotic motion cancellation. European Journal of Cardio-Thoracic Surgery 25 (2004) 786-790

4. Ortmaier, T.J.: Motion Compensation in Minimally Invasive Robotic Surgery. PhD thesis, Technische Universität München (2003) http://tumb1.biblio.tumuenchen. de/publ/diss/ei/2003/ortmaier.html.

5. Thakral, A., Wallace, J., Tomlin, D., Seth, N., Thakor, N.V.: Surgical motion adaptive robotic technology (s.m.a.r.t.): Taking the motion out of physiological motion. In: Proc. of the 4th Int. Conf. on Medical Image Computing and Computer-Assisted Intervention (MICCAI), Utrecht, The Netherlands (2001) 317-325

6. Bamieh, B., Giarr, L.: Identication of linear parameter varying models. In: Proc. 38'th IEEE Conference on Decision and Control. (1999) 